\title{
KNOWLEDGE DISCOVERY FOR PERVASIVE AND REAL-TIME INTELLIGENT DECISION SUPPORT IN INTENSIVE CARE MEDICINE
}

\author{
Filipe Portela ${ }^{1}$, Pedro Gago ${ }^{2}$, Manuel Filipe Santos ${ }^{1}$, Alvaro Silva $^{3}$, Fernando Rua ${ }^{3}$, \\ José Machado $^{4}$, António Abelha ${ }^{4}$, José Neves ${ }^{4}$ \\ ${ }^{l}$ Centro Algoritmi, Universidade do Minho, Guimarães, Portugal \\ ${ }_{2}^{2}$ Instituto Politécnico de Leiria, Leiria, Portugal \\ ${ }^{3}$ Unidade de Cuidados Intesnivos, Centro Hospitalar do Porto, Porto, Portugal \\ ${ }^{4}$ Departamento de Informática,Universidade do Minho, Azurem, Guimarães, Portugal \\ ${ }^{1}\{c f p, m f s\} @ d s i . u m i n h o . p t,{ }^{2}$ pgago@estg.ipleiria.pt, ${ }^{3}$ moreirasilva@clix.pt, \\ 3fernandorua.sci@hgsa.min-saude.pt, ${ }^{4}$ \{jmac,aabelha,jneves\}@di.uminho.pt
}

Keywords: Pervasive, Decision Support Process, Real-Time, Clinical Data, Data Acquisition, Intensive Care Medicine

\begin{abstract}
Pervasiveness, real-time and online processing are important requirements included in the researchers' agenda for the development of future generation of Intelligent Decision Support Systems (IDSS). In particular, knowledge discovery based IDSS operating in critical environments such of intensive care, should be adapted to those new requests. This paper introduces the way how INTCare, an IDSS developed in the intensive care unit of the Centro Hospitalar do Porto, will accommodate the new functionalities. Solutions are proposed for the most important constraints, e.g., paper based data, missing values, values outof-range, data integration, data quality. The benefits and limitations of the approach are discussed.
\end{abstract}

\section{INTRODUCTION}

In the future, the decision-making process and the form how the people make decisions should take into account new requirements like pervasiveness, real-time and online processing. This paper explores how these requirements can be integrated in the critical health care arena in order to improve the decision process. The work is part of a major project: INTCare - an IDSS for intensive care units.

The Intensive Care Units (ICU) are considered critical units where each decision needs to be correct and performed very carefully. The existence of a high number of data sources difficult, the data dissemination and the decision making process by the doctor. In the ICUs exist many electronic platforms with patient information, systems which collect vital signs and other systems that contains lab results, medical proceedings, etc., however still exist much information that were manually registered in the paper format. This is a common problem on the ICUs and is essentially associated to the nursing records (Lyerla, LeRouge, Cooke, Turpin, \& Wilson, 2010). The creation of intelligent agents (De Turck et al., 2007; Wooldridge, 1999) allow, perform some automatic tasks, continuously and in real-time. However the process automation has some restrictions, due to problems that arise during tasks execution like incorrect values, null values, missing identification and others. In order to resolve these problems we develop a platform for monitoring the patient data electronically with a total control of values by the humans. The main goal of this paper is to present architecture to ensure that all the clinical data that normally is used in the decisionmaking process is accessible electronically, enabling a pervasive and real-time approach. Beyond this chapter, a background will be presented on the ICU, the data acquisition process and the major problems detected. Chapter IV presents the platform developed in order obtain the decision variables in real-time and make them available in electronic and in online mode. Chapter V is dedicated to the data quality, and presents some forms of data processing to increase the data quality. Chapter VI makes a brief presentation of the intelligent agents that were used in the KD process. Finally, the results related to the data quality will be presented and discussed. Conclusions and future work cease the paper.

\section{BACKGROUND}

\subsection{Intensive Medicine Environment}

Intensive Medicine (IM) is a critical area where anything can fail, because the itensivists are dealing with human lives in particularly and weakened 
conditions. ICU uses IM to treat their patients and is a critical environment because have some complex health care situations (Bricon-Souf \& Newman, 2007), the activities occurring in it are sometimes adverse, dangerous and tiring the various organ systems of the patient may be affected at the same time (Apostolakos \& Papadakos, 2001) and this is a challenging for the that operate in this environment.

\subsection{INTCare}

INTCare is an IDSS that was developed to Intensive Medicine, with the main goal predicts the organ failure and outcome (Gago et al., 2006; Manuel Filipe. Santos et al., 2011) in real time (Portela et al., 2010). The system is being concluded and is been tested in the ICU of the Hospital Santo António (HSA) in Porto. The INTCare system is divided into four subsystems: data acquisition, knowledge management, inference and interface (Portela, Santos, et al., 2010) and uses intelligent agents to, for example, automate the collection, processing and transformation of data, and update the predictive models in real-time, without the human intervention.

\subsubsection{System Features}

The number of INTCare features has been increasing according new problems or situation that we find and with the objective to create a most complete system possible. In order to complete the KDD process, the system attends some requirements (Manuel Filipe Santos \& Portela, 2011): Online Learning; Real-Time; Adaptability; Data mining models; Decision models; Optimization; Intelligent agents; Pervasive; Accuracy; Safety; Privacy; Secure Access from Exterior; User Policy.

\subsubsection{System Requirements}

In order to accomplish the features defined before, the system has to have some important requirements (Portela, Santos, et al., 2010). These requirements were now incorporated and are part of KDD process: 1) Fault tolerance capacities; 2) Remove null and noisy data; 3) Ensure the patient identification; 4) Continuous data acquisition; 5) Time restrictions for the data acquisition and storage; 6) Digital data archive in order to promote the dematerialization of paper based processes; This paper will explain the decisions and alterations performed.

\subsection{Intelligent Agents}

The intelligent agents used by INTCare and are capable to performs autonomous actions, without the human interaction, in order to meet its goals (Gago, et al., 2006). To implement this system, use of multiagent systems is fundamental, with this is possible have various agents cooperates in order to manage a variety of problems (Foster, McGregor, \& El-Masri, 2005; Machado, 2006). INTCare uses intelligent agents to perform some actions that make the system work through autonomous actions that perform some essential tasks. These tasks support some system modules: Data Acquisition, Management of Knowledge, Inference and Interface. The flexibility and effectiveness of such systems depend on the agents and the interactions between them.

\subsection{Pervasive Health Care}

Pervasive HealthCare (PHC) appears with the objective to resolve some problems in the Health Care like the data quality and the data access. Varshney (U. Varshney, 2007, 2009) defines PHC as Health for all, anytime and anywhere by removing restrictions such as location and time, increasing the coverage and quality of health care. However the problem is how we can do it? Is it viable in a critical environment, like is Intensive Care? These questions arises, for though the PHC have the potential to reduce costs, improve service quality and facilitate the treatment of the patients also faces many technical hurdles and administrative (Upkar Varshney, 2003) as resistance to change and significant changes in systems and technologies.

The problem with this type of scenario is that the information is not always available when it is required that sometimes prevents the physician to make the best decision for the patient, this happens because there also a lot of information in paper. Solution arises as the possibility of providing all necessary information electronically and the creations of decision models that help the doctor make the best decision in real time.

\section{ICU - DATA ACQUISITION}

Data acquisition is a central issue for a pervasive intelligent decision support in order to enable a way of work in real-time. A considerable number of different data sources exist that should be integrated in the ICU data acquisition process.

\subsection{Data Sources}

This processed has essentiality in five data sources:

Vital Signs: Contains all vital signs collected by the sensors connected to the patient. This data are collect by the bedside monitors, e.g. Blood Pressure, SPO2, Respiratory and Frequency Rate, etc. 
Electronic Health Record (EHR): Has all information about the patient at admission e.g. patient identification (PID), age, name, sex, admission from, admission type, comorbidities, etc.

Drugs System: This system is responsible to control the patient therapeutic plan. For each patient, it contains a detailed plan with, the drugs prescription, dosages, administration dates, etc.

Lab Results: Every day patients make lab analysis the results will be collected and processed by a laboratory. It contains all the values for each patient: oximetry, clinical chemistry, and hematology.

Electronic Nursing Record (ENR): Allow to have all information about the patient admitted in the ICU in electronic format e.g. fluid balance, medical scores, therapeutics plans, events, etc.

\subsection{Data Acquisition Process}

The Data acquisition process has been modified in last two years. In the start (2009) more than $80 \%$ of data were register in paper or were not accessible electronically, of these, only $30 \%$ were stored offline (manually) in database.

Now in 2011 we can have all data in electronic format, and these, can be registered, validated or consulted in real-time and automatic or manual form. After we collect the data, the processing and transformation process begin; largely of this tasks will perform automatic by the intelligent agents.

Like we can see in Figure 1 are four, the data sources that contain information to the knowledge process, this data will be acquired and processed according each agent task. Finally this process is responsible for the processing and transformation data to the INTCare system according the variables required to the data mining. The main objective of autonomous acquisition process is processing the patient data in the moment that were collected and validate it according the values defined in ICU.

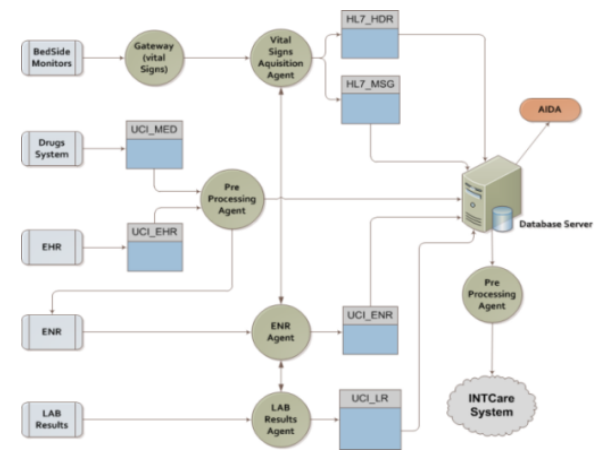

Figure 1: ICU Data Acquisition architecture

This operation will increase the data quality stored in the database, allow the calculation of critical events and prepares the data to create the best decision models.

\subsection{Data Acquisition Problems}

The number of problems associated to automatic data acquisition process is high, complex and can compromise all decision making process. These problems can have different natures, like, humans, techniques, informatics, environments, etc.

The most common problems are: sensors disconnected, data collected without PID, missing values and values with noise and system failures. In order to mitigate this problems, were defined the requirements that need to be addicted to the system.

\section{NURSING RECORDS - ENR}

After made a questionnaire we concluded that normally the decision is based in the vital-signs results, the registers presented in the Paper Nursing Record (PNR). Normally, proceedings, therapeutics, and events are also used in the decision models; however some of these data was not available in real-time. Moreover different platforms and formats should be considered. The decision process is not made instantly, this limitation stems from problems like the data access time, different platforms with the necessary data and the format how the data are stored, i.e., the data aren't clear (pdf format, difficult to analyse the entire hospital stay, dubious values).

Paper based registers, and the acquisition of wrong values is the main problems of the ICU and is the biggest obstacle for the creation of prevision and decision models. Based on the information collected and on a selection of the most important variables for the decision making process, a platform was created to allow the automatic data monitoring. PNR has been substituted by the ENR. ENR is a touchscreen system interoperating with all the information systems used in the ICU. Contains patients' data and allows for a higher number of functionalities to record, store and validate the values collected in the environment, in an online and real-time way.

\subsection{Systems Interoperability}

ENR encompasses a lot of data provided from other systems: EHR, Medical Systems, BM and Lab Results. This facilitates the nurses and doctor work, because now they know where is the information that they need to care the patient. With this option there are no longer islands of information, passing all the clinical and patient data to be accessible in the same space. 


\subsection{Main Features}

Besides the integration some other features are available with the introduction of ENR in the ICU. In order to enable the KDD process, the ENR contains a set of features to streamline the process:

- Collect information about patient process;

- Get correct vital signs values, therapeutic and drugs plans, Lab Results and medical request;

- Allow manual fluid balance registers, manual medical and nursing recording and validation of values and record of adverse events;

- Automatic calculation of medical scores;

- Store all validated data in database.

\subsubsection{Patient Clinical Data Logbook}

An important benefit in the use of this platform is the mode the clinical data are collected and presented for each day. The ENR is based on a grid divided in 24 hours, presenting for each hour the values of the selected variables, e.g., vital signs, drugs and schedules events. All monitoring data that are scheduled to some variables appear in the ENR automatically. The data will be collected and inserted by the agents. this data can be also stored manually by the nurses, near the patient, in the moment that the things happen. The nurse chooses the event that happened, from a set of predefined events, in that moment and this automatically appear in another table with the description and the time of the event. For the decision process, the medical staff can consult the patient historical registers, only choosing the date before. The base of the grid is the same, changing only the values and variables collected in the chosen day.

\subsubsection{Lab Results (LR)}

The Lab Results are not under the nurse's control in the ICU, because they are ordered by the doctors and executed by the labs. The clinical results appear in the ENR according to the laboratory work. This platform has a singular form to present the lab results in a grid. With this solution is possible to compare all the results by patient grouped by the clinical exam class and date. This facilitates the decision process because it makes possible the analyses of the results evolution by patient and by variable during all stay.

\subsubsection{ICU Scores}

In the ICU is used a set of scores, especially pain scores, Glasgow (Jones, 1979), SOFA (Vincent et al., 1998) and SAPS (Le Gall, Lemeshow, \& Saulnier, 1993) to predict some clinical problem or know the real situation of the patient. The introduction of ENR associated with the electronic acquisition and registering of all data, allow for the automatic scores calculation.

A mechanism of calculation was developed in the ENR for each target score. Every time a new value arrives to the variables included in the score, the ENR Agent verifies which the worst value of the day is and according to the range of each target score it assigns a punctuation to be calculated in the final. The final result only will be calculated when all variables of the score were obtained. If some variable wasn't collected automatically, the nurses can introduce the result manually. The calculation of scores will be explained in the next chapter.

\section{ICU - DATA QUALITY}

The quality of data is very important for a good decision-making process. The data quality interferes directly in the success of to the knowledge discovery process that occurs in the ICU. This process starts with the data stored in database collected from the Vital Signs, EHR, Drugs System, Lab Results and Events. All information collected will be stored in database. Dependent of the variables collected and the targets defined, the data will be validated and prepared according the processing and transformation rules.

The data resulting of this process is the real data collected in ICU about the patient. This data will be available through ENR and is prepared to be used by DM techniques (Manuel Filipe Santos \& Portela, 2011; Villas Boas et al., 2010) and create knowledge to the INTCare System. This process is represented in the Figure 2. The ENR collects the data that was automatic acquired, stored in database, processed and transformed. The resulting data from the processing tasks will be the real data and are these that will be showed by ENR. The ENR also is a data sources and also is subject to this process of quality.

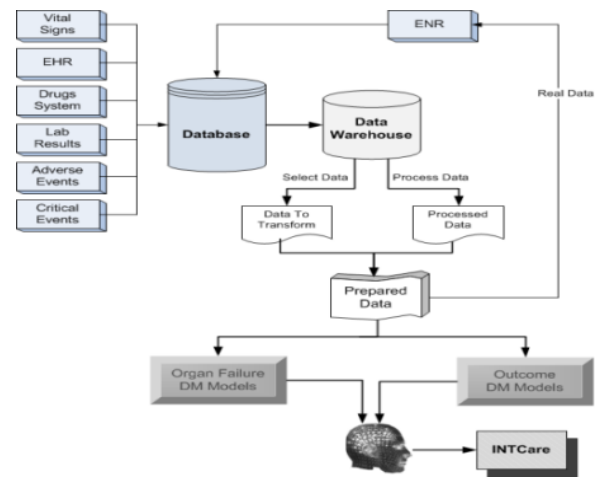

Figure 2: Knowledge Discovery Process in ICU 


\subsection{Data Processing Process}

All data collected will be processed by the agents that execute the tasks and rules defined for each variable / source. The data processing process is part of a set of INTCare requirements:

\section{a) Fault tolerance capacities and continuous data acquisition process}

For each value collected, system working or record expected, the agent will process the information received in database and if anything not in agreement with the expected, a message will be sent. Depending the type of fail, exist some predefined solution tasks: If the failure was in gateway, the agent send a message to the gateway and it will restart instantly, if the failure was in the LR or in drugs system a force refresh of the system will be done, finally if something fail in ENR system, the last record stored in database will be loaded.

\section{b) Processing to remove null and noisy data}

This process will be constantly running and has two types of operations. The first type consist in a constant looking for null records, if the line stored in database contains null values, this will be delete. The second type is responsible for autonomous validation of the data collected automatic and in real time from bedside monitors (BM) according the range values defined to ICU, i.e. if the vital signs values collected were correct and were possible in the ICU environment the agent will validate the results and store the data in a table with the real data. Table 1 shows the minimum and maximum values attributed to the vital signs variables that will be used to Data Mining. The objective is eliminating the data that has noise, or incorrect values. An example of noise values is the temperature, because when the sensor is disconnected from the patient the temperature collected is from the environment $\left(\sim 24^{\circ}\right.$ to $\left.27^{\circ}\right)$ and not from the patient $\left(\sim 35^{\circ}\right.$ to $\left.42^{\circ}\right)$. The final validation will be always done hourly by the nursing staff through the ENR functionalities. With this option if a value is out of range but is true, the nurse can rectify that and make that value real, registering this in the database.

Table 1: ICU Vital Signs Range.

\begin{tabular}{|c|c|c|}
\hline Vital Sign & Min & Max \\
\hline Blood Pressure (BP) & 0 & 300 \\
\hline SPO2 & 40 & 100 \\
\hline Temperature (Temp) & 30 & 45 \\
\hline Respiratory Rate (RR) & 0 & 40 \\
\hline Heart Rate (HR) & 0 & 250 \\
\hline
\end{tabular}

\section{c) Ensure the patient identification in records}

Put the patient id in the monitors, is a manual process, and because of that is a process that are in constant fails. The objective is tried to turn this process autonomous, without any type of human intervention. The first solution founded was put a RFID tag in the patient that will identify them in the system, but this was an expensive solution and slow to implement. In order to resolve this problem a more fast possible we decide create a task that verify, in the EHR, the patients admitted to ICU and have a null date of discharge then, it sees the number of the patient bed that came in the HL7 message sent by gateway and compares with the values present in the patient admission EHR table. After find a match, the pre-processing agent will substitute the in the message the null PID by the patient id present in the EHR. This make it an autonomous process, which runs every moments that arrives a new message to the table, it is a before insert operation.

\section{d) Time restrictions for the data acquisition and storage}

With the objective that data processing will be done with the most recent data acquired, a set of auto operations is scheduled, tasks like the auto save, auto refresh, auto validation of data, etc. If any operation can't be executed and if the agents don't receive any type of data to process and the responsible agent will send an alert message identifying the problem and possible solutions. Each individual agent (vital signs acquisition, preprocessing agent, ENR, and Lab Results) is responsible for the operation of their tasks group.

\section{e) Digital data archive in order to promote the dematerialization of paper based processes}

The solution founded to this requirement involves a set of agents, tasks and electronic process that can be executed automatic and controlled by the humans. The objective of this requirement is processing all Fluid balance, nursing records, medical scores, according the panel tables defined in ICU with this we hope ensure the actuality of tables, ensure that the data will be processed to data mining models i.e., all manual register types will be transformed in online and electronic records that can be done in real time by the humans in the moment that the situation happens or value need to be collected. This requirement was the base of development of the entire ENR, intelligent agents, interoperability between systems and other types of operations. 


\section{2 Data Transformation Process}

To the development of prevision models in real-time using Data Mining (DM) techniques and basing that in the offline results obtained in the past (Gago, Silva, \& Santos, 2007; Á. Silva, Cortez, Santos, Gomes, \& Neves, 2008) is necessary implement a set of automatic operations that facilitate the calculation of critical events and medical scores.

\section{a) Critical Events}

A critical event happened when a patient have values out of normal range for a determinate time. Table 2 is the base to the calculation of critical Events function and presents three types for the calculation of critical events. The calculation of critical events is a consequent task of the processing to remove null and noisy data. For this, this operations only will be used for the values prevalidated by the system, i.e., the calculation is done with base in the maximum range defined for ICU, the normal range and the time for an event be critical (A. Silva, Cortez, Santos, Gornesc, \& Neves, 2006).

First is verified if the value is normal or critical, next, will calculated the time of the event and stored in the critical events table, an event identification.

Table 2: ICU Critical Events Range.

\begin{tabular}{|l|c|c|c|c|}
\hline & BP & O2 & HR & Urine \\
\hline Normal Range & $90-180$ & $\geq 90 \%$ & $60-120$ & $\geq 30$ \\
\hline Critical Ev. a & $\geq 1 \mathrm{~h}$ & $\geq 1 \mathrm{~h}$ & $\geq 1 \mathrm{~h}$ & $\geq 2 \mathrm{~h}$ \\
\hline Critical Ev. b & $\geq 1 \mathrm{~h}$ in $2 \mathrm{~h}$ & $\geq 1 \mathrm{~h}$ in $2 \mathrm{~h}$ & $\geq 1 \mathrm{~h}$ in $2 \mathrm{~h}$ & - \\
\hline Critical Ev. c & $<60$ & $<80$ & $<30 \mathrm{~V}>180$ & $\leq 10$ \\
\hline
\end{tabular}

After received a row in the database, the agent will read the variable id, and will compare the result collected with the values present in Table 2 for that variable. If the value is out of normal range the row will be update and the type of result will be defined with critical (1) if the values is out of normal range and are worse than values defined for the critical event c, the row will be assigned to a critical event, will be update with number 2 and will be copied to table ICU_CEVENTS. The function 3 shows how the data collected will be tagged according the critical state $(0,1,2)$. To calculate the other two types of critical events will be used the sum functions to calculate the time that a value was out of normal range.

\section{b) Medical Scores}

There exist three types of scores that is used in ICU: MEWS (Gardner-Thorpe, Love, Wrightson, Walsh,
\& Keeling, 2006), SAPS (Le Gall, et al., 1993), SOFA (Vincent, et al., 1998). For each score a set of transformations operation will be done. The operations are similar for all scores. The SOFA scores is used in DM models and will presented that transformation. The value is allocated according to the score punctuation table. Using the number of points associated, the final score will be calculated and the result will be inserted in the scores table. The results obtained are collected automatically.

Table 3: SOFA Table (respiratory organ)

\begin{tabular}{|c|c|c|}
\hline System & Variable to the Calculation & Score \\
\hline \multirow{5}{*}{$\begin{array}{l}\text { Respiratory } \\
\text { System }\end{array}$} & \multicolumn{2}{|l|}{$\mathrm{PaO}_{2} / \mathrm{FiO}_{2}(\mathrm{mmHg})$} \\
\hline & $<400$ & 1 \\
\hline & $<300$ & 2 \\
\hline & < 200 and mechanically ventilated & 3 \\
\hline & < 100 and mechanically ventilated & 4 \\
\hline
\end{tabular}

\section{ICU - AGENTS}

In an ICU data can be acquired from a different number of sources. In our architecture we propose several data acquisition agents with clear boundaries and responsibilities. Moreover, given the paramount importance of data quality for data mining a data quality agent responsible for detecting errors in the data was devised. Indeed, data acquisition in the ICU is error prone as, for instance, sensors may be displaced as patients move. The agents included in the data acquisition module are:

A Gateway agent $\left(a_{\text {gat }}\right)$ connected to the bedside monitors. Operating in real-time, this agent captures the vital signs data from the bedside monitors (BM).

Vital Signs Acquisition agent is in charge of receiving and parsing the HL7 messages sent by the Gateway. Other than that real time parsing this agent also stores the information in the database tables.

In order to allow a straightforward data entry by the nurses, the ENR Agent $\left(a_{\text {enr }}\right)$ was created (Portela, Vilas-Boas, Santos, \& Fernando, 2010). This agent is able to save the data automatically or as a response to a request from the nurse. With this agent we are able to capture the clinical data from the medical and nursing staff. The data recorded by this agent includes the fluid balance, patient proceedings, and other scores.

Working in close connection with the ENR agent, the $\mathbf{L R}$ agent $\left(\mathrm{a}_{l}\right)$ is responsible for capturing the clinical data from the lab results. Every five minutes this agent checks if there are new lab results available. Whenever there are new lab results available the $\mathbf{L R}$ agent gets them and stores the information in the database before sending the relevant information to the ENR agent. If we are to 
use the collected data for decision support we must make as sure as possible that it contains no errors. In INTCARE architecture this is a task of the PreProcessing $\left(a_{p p}\right)$ agent.

In an ICU setting it is easy to see sensors that aren't correctly placed. This happens mostly due to patient movement, even if incorrectly placed most sensors continue to collect data. Naturally this means that some pre-processing is necessary in order to avoid that such incorrect values reach the records. Whenever this happens for a short period the preprocessing agent replaces the incorrect values with the average of the values collected prior and after the error. If the error condition lasts for a long time the agent replaces the values with zeros, indicating that no valid values were collected. Finally, this agent creates a valid medical record for the patient. It does this by combining all the available values and ensuring that those values make sense.

\section{RESULTS}

Until 2009 the doctors based their decisions on information essentially in paper format or on data that were accessible only in specific systems or places. Nowadays, all of this information is available in electronic format and can be accessed online and in real-time, in a pervasive approach.

Table 4 shows the evolution of data sources and the data access verified between 2009 and 2011. The data source can be Paper (P), Electronic (E) or both $(\mathrm{P} / \mathrm{E})$, i.e. some information are in paper and others are in digital format. The chart 1 presents a visualization of the percentage of data in each of formats for those two years. Like we can verify in $2011,100 \%$ of the data are electronic, acquired in real-time and available online. This chart also presents the percentage of processes without patient ID (PID), that represents a decrease from $53 \%$ in 2009 to $0 \%$ in 2011 , i.e., now all messages received and data collected has PID.

Table 4: ICU Data Sources Format.

\begin{tabular}{|l|c|c|c|c|}
\hline \multirow{2}{*}{$\begin{array}{c}\text { Data Sources } \\
\text { Variables }\end{array}$} & \multicolumn{2}{|c|}{2009} & \multicolumn{2}{c|}{2011} \\
\cline { 2 - 5 } & Source & Online & Source & Online \\
\hline Nursing & $\mathbf{P}$ & $\mathbf{x}$ & $\mathbf{E}$ & $\mathbf{V}$ \\
\hline Vital Signs & $\mathbf{P}$ & $\mathbf{x}$ & $\mathbf{E}$ & $\mathbf{V}$ \\
\hline Drug System & $\mathbf{P} / \mathbf{E}$ & $\mathbf{V}$ & $\mathbf{E}$ & $\mathbf{V}$ \\
\hline Patient EHR & $\mathbf{E}$ & $\mathbf{V}$ & $\mathbf{E}$ & $\mathbf{V}$ \\
\hline Fluid balance & $\mathbf{P}$ & $\mathbf{x}$ & $\mathbf{E}$ & $\mathbf{V}$ \\
\hline Procedures & $\mathbf{P} / \mathbf{E}$ & $\mathbf{x}$ & $\mathbf{E}$ & $\mathbf{V}$ \\
\hline Lab Results & $\mathbf{P}$ & $\mathbf{x}$ & $\mathbf{E}$ & $\mathbf{V}$ \\
\hline Patient Events & $\mathbf{P} / \mathbf{E}$ & $\mathbf{x}$ & $\mathbf{E}$ & $\mathbf{V}$ \\
\hline Ventilations & $\mathbf{P}$ & $\mathbf{x}$ & $\mathbf{E}$ & $\mathbf{V}$ \\
\hline Patient Scores & $\mathbf{P}$ & $\mathbf{x}$ & $\mathbf{E}$ & $\mathbf{V}$ \\
\hline ICU Scores & $\mathbf{P}$ & $\boldsymbol{*}$ & $\mathbf{E}$ & $\mathbf{V}$ \\
\hline
\end{tabular}

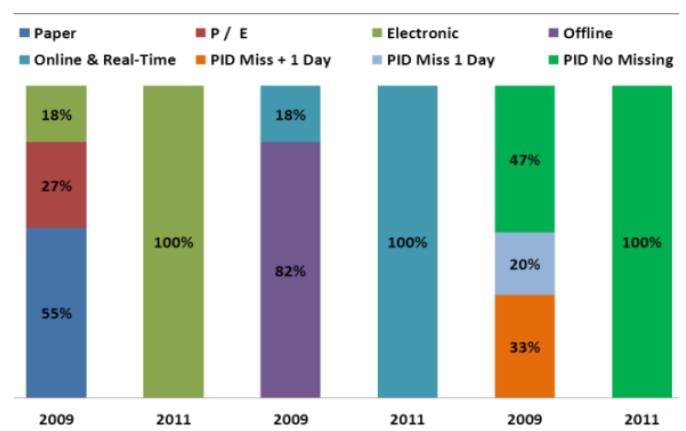

Chart 1: Data access Formats (\%)

The modifications introduced in the ICU information systems and the implementation of data processing tasks, the level of the data quality increased significantly. Analyzing the example in chart 2, we achieved benefits in the all variables presented, especially in the heart rate where more than $75 \%$ were worst values, i.e., values that were collected and were out of normal range predefined in the ICU. The overall benefit is about $34 \%$ which represents the volume of data collected ignored after the pre-processing.

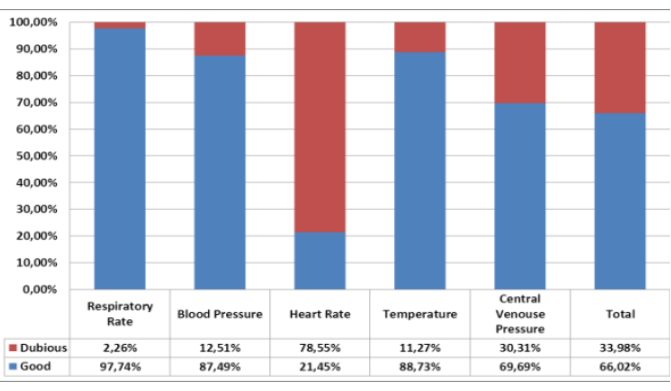

Chart 2: Data Ouality (\%)

\section{CONCLUSIONS AND FUTURE WORK}

This paper presented an approach to the KDD procedure in order to enable a pervasive, online and real-time processing of data in ICU. Such approach brought improvements in the information availability and consequently a more proactive attitude by the doctors is facilitated. The doctors are supported in their decisions anytime and anywhere. In particular, data quality problems were completely solved, e.g., monitored null values, values out of the range and wrong patient ID. Finally, the quality of decision making process has been significantly increased. All the data (100\%) used in the decision process and in data mining models are reliable, i.e. the values are in the range defined by ICU and the doctors don't deal with dubious values. In the future 
we will study the impact in the validity of data mining models adding the data (decision variables) obtained from this process, i.e. the therapeutics and procedures. In order to control the failures, a tolerance plan also will be created.

\section{ACKNOWLEDGEMENTS}

The authors would like to thank FCT (Foundation of Science and Technology, Portugal) for the financial support through the contract PTDC/EIA/72819/ 2006. The work of Filipe Portela was supported by the grant SFRH/BD/70156/2010 from FCT.

\section{REFERENCES}

Abelha, A., Machado, J., Santos, M., Allegro, S., Rua, F., Paiva, M., et al. Agency for Integration, Diffusion and Archive of Medical Information.

Apostolakos, M. J., \& Papadakos, P. J. (2001). The Intensive Care Manual: McGraw-Hill Professional.

Bricon-Souf, N., \& Newman, C. R. (2007). Context awareness in health care: A review. [10.1016/j.ijmedinf.2006.01.003]. International Journal of Medical Informatics, 76(1), 2-12.

De Turck, F., Decruyenaere, J., Thysebaert, P., Van Hoecke, S., Volckaert, B., Danneels, C., et al. (2007). Design of a flexible platform for execution of medical decision support agents in the intensive care unit. Computers in Biology and Medicine, 37(1), 97-112.

Foster, D., McGregor, C., \& El-Masri, S. (2005, 2005). A survey of agent-based intelligent decision support systems to support clinical management and research.

Gago, P., Santos, M. F., Silva, Á., Cortez, P., Neves, J., \& Gomes, L. (2006). INTCare: a knowledge discovery based intelligent decision support system for intensive care medicine. Journal of Decision Systems.

Gago, P., Silva, A., \& Santos, M. F. (2007, Dec 03-07). Adaptive decision support for intensive care. Paper presented at the 13th Portuguese Conference on Artificial Intelligence, Guimaraes, PORTUGAL.

Gardner-Thorpe, J., Love, N., Wrightson, J., Walsh, S., \& Keeling, N. (2006). The value of Modified Early Warning Score (MEWS) in surgical in-patients: a prospective observational study. Annals of The Royal College of Surgeons of England, 88(6), 571.

Jennings, N. R. (2000). On agent-based software engineering. Artificial Intelligence, 117(2), 277-296.

Jones, C. (1979). Glasgow coma scale. AJN The American Journal of Nursing, 79(9), 1551.

Le Gall, J. R., Lemeshow, S., \& Saulnier, F. (1993). A new Simplified Acute Physiology Score (SAPS II) based on a European/North American multicenter study. JAMA, 270(24), 2957-2963.
Lyerla, F., LeRouge, C., Cooke, D. A., Turpin, D., \& Wilson, L. (2010). A Nursing Clinical Decision Support System And Potential Predictors Of Head-Of-Bed Position For Patients Receiving Mechanical Ventilation. American Journal of Critical Care, 19(1), 39-47.

Machado, J. A., António. Santos, Manuel, Neves, José. (2006). Multi-agent based Problem Solving in Medical Decision Support Systems. In R. C. a. F. L. Vale Z. (Ed.), Decision Support, Knowledge and Decision Technologies. Porto: IPP, ISEP.

Portela, F., Santos, M., Vilas-Boas, M., Rua, F., Silva, Á., \& Neves, J. (2010). Real-time Intelligent decision support in intensive medicine. Paper presented at the KMIS 2010- International Conference on Knowledge Management and Information Sharing.

Portela, F., Vilas-Boas, M., Santos, M. F., \& Fernando, R. (2010). Improvements in data quality for decision support in Intensive Care. Paper presented at the eHealth 2010 - 3rd International ICST Conference on Electronic Healthcare for the 21st century.

Santos, M. F., \& Portela, F. (2011). Enabling Ubiquitous Data Mining in Intensive Care - Features selection and data pre-processing. Paper presented at the 13th ICEIS.

Santos, M. F., Portela, F., Vilas-Boas, M., Machado, J., Abelha, A., \& Neves, J. (2011). INTCARE - Multi-agent approach for real-time Intelligent Decision Support in Intensive Medicine. Paper presented at the 3rd International Conference on Agents and Artificial Intelligence (ICAART).

Silva, A., Cortez, P., Santos, M. F., Gornesc, L., \& Neves, J. (2006). Mortality assessment in intensive care units via adverse events using artificial neural networks. Artificial Intelligence in Medicine, 36(3), 223-234.

Silva, Á., Cortez, P., Santos, M. F., Gomes, L., \& Neves, J. (2008). Rating organ failure via adverse events using data mining in the intensive care unit. Artificial Intelligence in Medicine, 43(3), 179-193.

Varshney, U. (2003). Pervasive Healthcare. Computer, $36(12), 138-140$.

Varshney, U. (2007). Pervasive healthcare and wireless health monitoring. Mobile Networks and Applications, 12(2), 113-127.

Varshney, U. (2009). Pervasive Healthcare Computing: EMR/EHR, Wireless and Health Monitoring: SpringerVerlag New York Inc.

Villas Boas, M., Gago, P., Portela, F., Rua, F., Silva, Á., \& Santos, M. F. (2010). Distributed and real time Data Mining in the Intensive Care Unit. ECAI 2010.

Vincent, J., Mendonça, A., Cantraine, F., Moreno, R., Takala, J., Suter, P., et al. (1998). Use of the SOFA score to assess the incidence of organ dysfunction/failure in intensive care units : Results of a multicenter, prospective study. Critical care medicine, 26, 1793-1800.

Wooldridge, M. (1999). Intelligent agents Multiagent systems: a modern approach to distributed artificial intelligence (pp. 27-77): MIT Press. 